\title{
BMJ Open Healthcare costs in chronic kidney disease and renal replacement therapy: a population-based cohort study in Sweden
}

\author{
Jonas K Eriksson, ${ }^{1}$ Martin Neovius, ${ }^{1}$ Stefan $\mathrm{H}$ Jacobson, ${ }^{2}$ Carl-Gustaf Elinder, ${ }^{3,4}$
} Britta Hylander ${ }^{5}$

To cite: Eriksson JK Neovius M, Jacobson SH, et al. Healthcare costs in chronic kidney disease and renal replacement therapy: a population-based cohort study in Sweden. BMJ Open 2016;6:e012062.

doi:10.1136/bmjopen-2016012062

- Prepublication history and additional material is available. To view please visit the journal (http://dx.doi.org/ 10.1136/bmjopen-2016012062).

Received 28 March 2016 Revised 1 August 2016 Accepted 2 September 2016

CrossMark

For numbered affiliations see end of article.

Correspondence to Dr Jonas Eriksson; jonas.eriksson@ki.se

\section{ABSTRACT}

Objective: To compare healthcare costs in chronic kidney disease (CKD) stage 4 or 5 not on dialysis (estimated glomerular filtration rate $<30 \mathrm{~mL} / \mathrm{min} /$ $1.73 \mathrm{~m}^{2}$ ), peritoneal dialysis, haemodialysis and in transplanted patients with matched general population comparators.

Design: Population-based cohort study.

Setting: Swedish national healthcare system.

Participants: Prevalent adult patients with CKD 4 or 5 $(n=1046$, mean age 68 years), on peritoneal dialysis $(n=101 ; 64$ years), on haemodialysis ( $n=460 ; 65$ years) and with renal transplants ( $\mathrm{n}=825 ; 52$ years) were identified in Stockholm County clinical quality registers for renal disease on 1 January 2010. 5 general population comparators from the same county were matched to each patient by age, sex and index year.

\section{Primary and secondary outcome measures:} Annual healthcare costs in 2009 incurred through inpatient and hospital-based outpatient care and dispensed prescription drugs ascertained from nationwide healthcare registers. Secondary outcomes were annual number of hospital days and outpatient care visits.

Results: Patients on haemodialysis had the highest mean annual cost (€87 600), which was 1.49 $(95 \% \mathrm{Cl} 1.38$ to 1.60$)$ times that observed in peritoneal dialysis ( $€ 58600)$. The mean annual cost was considerably lower in transplanted patients $(€ 15500)$ and in the CKD group ( $€ 9600)$. In patients on haemodialysis, outpatient care costs made up more than two-thirds ( $€ 62500)$ of the total, while costs related to fluids (\$29 900) was the largest cost component in patients on peritoneal dialysis $(51 \%)$. Compared with their matched general population comparators, the mean annual cost $(95 \% \mathrm{Cl})$ in patients on haemodialysis, peritoneal dialysis, transplanted patients and patients with CKD was 45 (39 to 51), 29 (22 to 37), 11 (10 to 13) and 4.0 (3.6 to 4.5$)$ times higher, respectively.

Conclusions: The mean annual costs were $~ 50 \%$ higher in patients on haemodialysis than in those on peritoneal dialysis. Compared with the general population, costs were substantially elevated in all groups, from 4-fold in patients with CKD to 11, 29
Strengths and limitations of this study

- In this population-based study, data were collected from routine clinical care to which there is universal access in Sweden.

- By linking nationwide healthcare registers using the personal identity number, follow-up on an individual level was virtually complete regarding hospital days, hospital-based outpatient care and dispensed prescription drugs.

- Costs related to in-hospital use of erythropoietinstimulating agents for patients on haemodialysis were assessed using records from a smaller patient sample.

- Costs related to fluids for patients on peritoneal dialysis were assessed using aggregated data, since these were not collected on an individual level in registers.

- Although the proportion of undiagnosed individuals with chronic kidney disease stage 4 or 5 has decreased with time, this group is still underdiagnosed and an unknown number of these patients were missed.

and 45 times higher in transplanted patients and patients on peritoneal dialysis and haemodialysis, respectively.

\section{INTRODUCTION}

Chronic kidney disease (CKD) and end-stage renal disease are associated with substantial healthcare resource use and considerably higher mortality. ${ }^{1}{ }^{2}$ Although some reports suggest a decline in the incidence of $\mathrm{CKD},{ }^{3}$ demographic changes together with increasing type 2 diabetes are likely to result in a higher prevalence of CKD. ${ }^{4}$ To evaluate the value of therapeutic interventions in this patient group, an assessment of the economic burden related to CKD and end-stage renal disease is necessary, as is an available 
benchmark in terms of the corresponding costs in the general population.

Several studies have investigated the burden or costs in patients at different stages of CKD and renal replacement therapy, using different methods and in different settings. ${ }^{5-7}$ The majority of cost studies from Europe and North America of patients on dialysis have reported higher healthcare costs of haemodialysis ranging from 1.0 to 1.9 times the cost in peritoneal dialysis. ${ }^{5}$ Similarly, previous studies have reported increasing healthcare use and costs with higher CKD stages, ${ }^{8-11}$ and estimates in patients with CKD that are 2-3 times the cost as compared with controls without CKD. ${ }^{9}{ }^{11}$ Recent studies from Europe, North America and Australia on the burden of CKD and renal replacement therapy with reported annual per patient cost have typically been based on small study samples or by using a modelling approach collecting data from published aggregated estimates. ${ }^{12-21}$ Only a few studies have used individual data from larger study samples. ${ }^{8-10}$

To the best of our knowledge, no study from the same study population on an individual level has described healthcare use and costs in CKD stages 4 or 5 on dialysis, haemodialysis, peritoneal dialysis or transplanted patients separately, nor has it compared the result to costs in the general population.

The aim of this population-based cohort study was to examine annual costs assessed from Swedish nationwide healthcare registers related to hospital days, outpatient care visits and prescription of drugs in prevalent CKD stage 4 or 5 not on dialysis, peritoneal dialysis, haemodialysis and transplanted patients, and to put these costs in relation to matched general population comparators.

\section{METHODS}

In this population-based cohort study, we identified patients in CKD stage 4 or 5 not on dialysis, patients on dialysis and transplanted patients from clinical quality of care registers in Stockholm County and added data from national health registers. By using the personal identity number, a unique number assigned to all Swedish residents, ${ }^{22}$ we enriched these data with inpatient and hospital-based outpatient care from the National Patient Register as well as data on dispensed drugs from the Prescribed Drug Register kept by the National Board of Health and Welfare.

\section{CKD and the Swedish National Health Service}

Sweden had a population of 7.4 million $\geq 18$ years on 31 December 2009 (http://www.scb.se) and comprised 21 counties. Stockholm County was the biggest with 1.6 million inhabitants $\geq 18$ years, accounting for $22 \%$ of the population. The Swedish healthcare system was tax funded and offered universal access, while prescription drugs were provided free of charge above a threshold of around $€ 200$ annually.
Patients with renal replacement therapy were treated by nephrologists in inpatient and outpatient facilities, ${ }^{23}$ rather than by general practitioners, while care for patients with CKD was a mix of mainly hospital-based outpatient care and, to some extent, primary care. The decision to initiate renal replacement therapy was made by nephrologists from clinical evaluations based on the Swedish guidelines ${ }^{24}$ originating from the National Kidney Foundation-Kidney Disease Outcomes Quality Initiative (NKF-K/DOQI) guidelines ${ }^{25}$ and the corresponding European guidelines. ${ }^{26}$

\section{Identification of patients in quality register sources Patients with CKD}

From the Stockholm County CKD Register, we identified adult prevalent patients with CKD in stage 4 or 5 who were not on dialysis on 1 January 2010 and registered at Karolinska and Danderyd University Hospital in the outpatient setting. This does not include all patients in CKD stages 4 and 5 in the county, as some may get care elsewhere and some remain undetected. Furthermore, patients in CKD stages 1-3B were generally not registered and were for this reason not included in the study. Stages 4 and 5 were defined as an estimated glomerular filtration rate (eGFR) of $15-29.9$ and $<15$, respectively. GFR was estimated using the abbreviated Modification of Diet in Renal Disease equation (MDRD; $\mathrm{mL} / \mathrm{min} / 1.73 \mathrm{~m}^{2}$ ) using serum creatinine levels. ${ }^{27}$ Individuals with missing eGFR at baseline were excluded from CKD analyses $(n=5)$. Data on albuminuria were incomplete and therefore no analyses by albuminuria status were performed.

\section{Patients on renal replacement therapy}

Prevalent patients on hospital-based dialysis or with a kidney transplant on 1 January 2010 were identified in the Swedish Register of Renal Replacement Therapy, which includes all adult patients on renal replacement therapy in Stockholm County. ${ }^{28} 29$

\section{General population comparators from the Register of the Total Population}

From the Register of the Total Population held by Statistics Sweden, up to five general population comparators from Stockholm County were sampled and matched on age, sex and index year to each patient (exact matching). The matched comparators received the same index date as did their corresponding patient. Data on emigration and highest attained education were also retrieved from the Register of the Total Population.

\section{The National Patient Register}

Data on inpatient and hospital-based outpatient care were retrieved from the Swedish National Patient Register in 2009. This register contains the personal identity number, visit/admission date (and discharge date for the inpatient component), diagnostic-related group (DRG) associated with the visit, and main as well as contributory diagnoses coded according to the 
International Classification of Diseases V.10 (ICD-10). ${ }^{30}$ Comorbid conditions were defined as having a visit in inpatient or outpatient care during the past 10 years with a main or contributory diagnosis of the respective ICD codes used (see etable 1).

Hospital days and outpatient visits were converted into costs using the DRG coding system, where clinically similar hospitalisations or outpatient visits are grouped together. The price per DRG is fixed for a specific year, and is a weighted average for all the healthcare services delivered in that group and year. In 2009, the inpatient and outpatient components of the National Patient Register included around 580 and 400 DRG groups, respectively.

\section{The Prescribed Drug Register}

From the Prescribed Drug Register, we collected data on dispensed prescriptions in ambulatory care in 2009. Data on in-hospital drug use are not recorded on a patient level in the Prescribed Drug Register. Among other variables, the register includes the personal identity number, date of prescription and dispensation, costs (total cost, patient cost, reimbursed cost), dosage, route of administration, and name as well as the Anatomic Therapeutic Chemical (ATC) code of the drug.

\section{Outcome and follow-up}

The main outcome was annual healthcare costs incurred through inpatient and outpatient care, as well as dispensed prescription drugs. Secondary outcomes were annual number of hospital days and outpatient visits. Costs were assessed during 2009 and converted to euros $(€ 1=\mathrm{Kr} 9.54$ Swedish kronor in 2010). Patients were followed in the same health state as when they were identified in 1 January 2010, with estimated annualised costs in those patients who did not have 1 year of follow-up.

\section{Annualised costs}

Healthcare use and costs in patients who were not in CKD stage 4 or 5 , on dialysis, or did not yet have a kidney transplant on 1 January 2009, and hence were not followed the complete year of 2009 (all patients were identified on 1 January 2010), were annualised from the time of entry into the respective health states. To have enough data available for each patient, we restricted the study population to patients with at least 1 month of follow-up, that is, patients who were exposed on or before 1 December 2009. For example, if a patient started haemodialysis on 1 July 2009, we doubled that patient's healthcare use and costs to achieve an annualised estimate. For transplanted patients who received a kidney transplant in 2009, the cost for the kidney transplant procedure was included. However, costs related to the transplantation procedure were not annualised.

\section{Costs related to haemodialysis visits}

As a data quality control, patients on haemodialysis who had $<2$ registered dialysis visits per week $(n=135)$ in the
National Patient Register were scrutinised using the patient registration database at the clinic. In most of these patients, visits that were found in the clinical database and that for some reason were not registered in the National Patient Register were added. In total of 88 $(65 \%)$ of the scrutinised patients on haemodialysis, we added information from the registration database at the clinic. For a few patients $(n=17)$ with unreasonable few registered haemodialysis visits in the clinical database, visits were imputed. For patients having missing visit data for time periods $\geq 3$ months and where extrapolation was not possible due to few visits $(\mathrm{n}=14)$, the number of visits was imputed with 156 haemodialysis visits per year (3 visits/week). In three patients with missing data for time periods $<3$ months $(n=3)$, the number of visits per week was extrapolated over time for these short time periods.

\section{Costs related to ESA in haemodialysis and fluids in peritoneal dialysis}

Since the Prescribed Drug Register does not include in-hospital use of drugs, the cost of erythropoietinstimulating agents (ESA) is underestimated in patients on haemodialysis when using this data source. We retrieved data on ESA use in a subsample of patients on haemodialysis $(n=85)$ and calculated the cost using 2009 drug prices in Sweden (http://www.tlv.se). In this subsample, the annual cost per patient related to ESA was estimated at $€ 3911$, which we applied to all patients on haemodialysis. Further, the cost of fluids for peritoneal dialysis is also not covered by the Prescribed Drug Register. This cost has previously been estimated at Kr200 000-Kr370 000 (€21 000-€38 800) per patient and year. ${ }^{31}$ We used the mid-value (€29 900) in this range and applied it on all patients on peritoneal dialysis.

\section{Statistical analysis}

Cost distributions were non-normal in patients in CKD stage 4 or 5, patients on peritoneal dialysis and transplanted patients, but approached a normal distribution in patients on haemodialysis (figure 1). Since the arithmetic mean has been described to be the most informative measure for cost and resource use data, ${ }^{32}$ we report the mean annual cost (complemented by the median for descriptive purposes). When comparing costs in CKD stages 4 or 5 , peritoneal dialysis, haemodialysis and transplanted patients, and when comparing patients versus their matched general population comparators, annual cost ratios were adjusted for age, sex and diabetes status using a generalised linear regression model with negative binomial distribution, and 95\% CIs were estimated using robust SEs. In sensitivity analysis, we fitted a generalised linear model with $\log -\gamma$ distribution, as well as in addition to age, sex and diabetes status adjusted for malignancy, circulatory disease, hypertension, cardiovascular disease, myocardial infarction, stroke and chronic obstructive pulmonary disease (see etable 1).

Statistical analyses were performed using SAS (V.9.3). 

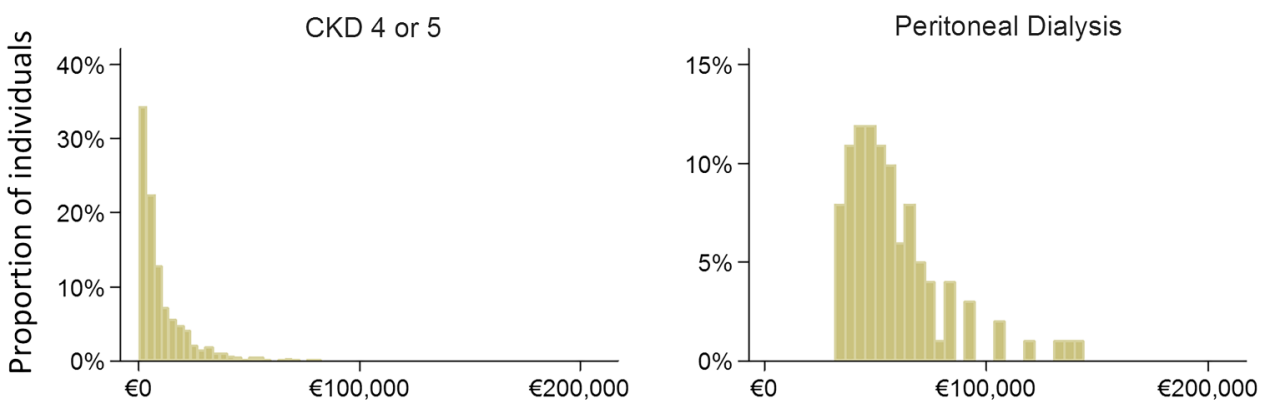

\section{RESULTS}

A total of 2432 prevalent patients who were alive on 1 January 2010 were included (table 1). Patients in CKD stage 4 or $5(n=1046)$ were on average aged 68 years, while patients on dialysis $(\mathrm{n}=101$ on peritoneal dialysis; $\mathrm{n}=460$ on haemodialysis) were younger ( 64 and 65 years, respectively), and transplanted patients $(n=825)$ much younger (52 years). All groups were predominantly male, and the education level was broadly similar to that in the general population (see table 1 , etable 2). The vast majority of transplanted patients had complete follow-up through 2009 (93\%), while one-quarter of the patients in CKD stage 4 or 5 and patients on haemodialysis did not have the full year of follow-up, and $40 \%$ of the patients in the peritoneal dialysis group were also followed for $<1$ year (table 1 ).

Regarding the selected register-identified comorbidities, $\sim 90 \%$ of the patients had circulatory disease history, with about $15 \%$ having had a myocardial infarction and $10 \%$ a stroke (except transplanted patients; figure 2). Patients in CKD stage 4 or 5 and patients on dialysis were similar, with the exception of a higher prevalence of cardiovascular disease in the group of patients on haemodialysis. The younger transplanted patients displayed lower prevalence, as compared with the patients with CKD and patients on dialysis, for most of the selected comorbidities, but a higher occurrence of malignancies. All groups had similar prevalence of chronic obstructive pulmonary disease as the general population comparators, but displayed higher or much higher prevalence in the other selected comorbidities, including diabetes where more than $30 \%$ of patients (except the transplanted group) had diabetes, compared with $6-9 \%$ in the matched general population (see etable 2).

\section{Total costs}

The highest mean annual cost was observed in the haemodialysis group with $€ 87600$, out of which $71 \%$ were accounted for by outpatient care costs ( $€ 62500$; figure 3; see etable 3). Of the total outpatient care cost, 97\% (€60 400) were costs for visits listing dialysis.

Patients on peritoneal dialysis incurred a mean annual cost of $€ 58600$ which was about two-thirds of the cost compared with patients on haemodialysis (adjusted ratio (haemodialysis vs peritoneal dialysis) $1.49,95 \%$ CI 1.38 to 1.60; table 3). The largest cost component in the peritoneal dialysis group was costs related to fluids (€29 900; $51 \%$ of total cost), while costs related to inpatient and outpatient care were similar (€11400 and $€ 10000$, respectively).

In contrast, transplanted patients was the only group where dispensed prescription drugs made up the largest cost component ( $€ 6800 ; 44 \%$ of total cost), while the mean annual cost in this group of $€ 15500$ was a fourth of the estimated cost in patients on peritoneal dialysis (adjusted ratio (peritoneal dialysis vs transplanted) 4.04, $95 \%$ CI 3.58 to 4.56 ; table 3 ).

Patients in CKD stage 4 or 5 displayed the lowest mean annual costs at $€ 9600$, where $45 \%$ (€4300) was accounted for by inpatient care. The adjusted mean annual cost ratio for transplanted versus CKD stage 4 or 5 was 1.70 (95\% CI 1.51 to 1.92 ; table 3 ). This difference was primarily explained by greater use of immunosuppressive drugs but also partly due to more expensive 
Table 1 Participant characteristics ${ }^{\star}$

\begin{tabular}{|c|c|c|c|c|}
\hline & $\begin{array}{l}\text { CKD stage } 4 \text { or } 5 \\
\text { (not on dialysis) }\end{array}$ & $\begin{array}{l}\text { Peritoneal } \\
\text { dialysis }\end{array}$ & Haemodialysis & Transplanted \\
\hline $\mathrm{N}$ & 1046 & 101 & 460 & 825 \\
\hline Sex (\% men) & $683(65)$ & $53(52)$ & $271(59)$ & $513(62)$ \\
\hline eGFR†, mean (SD) & $18(6)$ & - & - & - \\
\hline$<15$ & $338(32 \%)$ & - & - & - \\
\hline $15-29.9$ & $708(68 \%)$ & - & - & - \\
\hline \multicolumn{5}{|l|}{ Time since transplantation (years) } \\
\hline Mean (SD) & - & - & - & $9.1(7.5)$ \\
\hline Median (25th-75th) & - & - & - & $7.1(3.3-12.8)$ \\
\hline \multicolumn{5}{|l|}{ Age (years) } \\
\hline Mean (SD) & $68(14)$ & $64(16)$ & $65(15)$ & $52(14)$ \\
\hline Median (25th-75th) & $71(60-79)$ & $68(54-78)$ & $68(55-75)$ & $54(43-63)$ \\
\hline \multicolumn{5}{|l|}{ n (\%) } \\
\hline $18-49$ years & $130(12)$ & 19 (19) & $76(17)$ & $336(41)$ \\
\hline $50-59$ years & $126(12)$ & $16(16)$ & $71(15)$ & $212(26)$ \\
\hline $60-69$ years & $232(22)$ & $22(22)$ & $125(27)$ & $224(27)$ \\
\hline 70 years & $558(53)$ & $44(44)$ & $188(41)$ & $53(6)$ \\
\hline \multicolumn{5}{|l|}{ Education level¥ (\%) } \\
\hline Patients $<75$ years & $636(61)$ & $66(65)$ & $343(75)$ & $808(98)$ \\
\hline$\leq 9$ years & $172(27)$ & $14(21)$ & $115(34)$ & $173(21)$ \\
\hline $10-12$ years & $260(41)$ & $31(47)$ & $137(40)$ & $347(43)$ \\
\hline$>12$ years & $193(30)$ & $19(29)$ & 69 (20) & $278(34)$ \\
\hline Missing & $11(2)$ & $2(3)$ & $22(6)$ & $10(1)$ \\
\hline Patients $\geq 75$ years (no information) & $410(39)$ & $35(35)$ & $117(25)$ & $17(2)$ \\
\hline First registration in 2009 & $260(25 \%)$ & $40(40 \%)$ & $120(26 \%)$ & $57(7 \%)$ \\
\hline Mean (SD) & $323(89)$ & $288(113)$ & $320(90)$ & $353(52)$ \\
\hline Median (25th-75th centile) & $365(365-365)$ & $365(217-365)$ & $365(352-365)$ & $365(365-365)$ \\
\hline
\end{tabular}

inpatient care among transplanted patients (table 2, figure 3).

In a stratified analysis on treatment start before or during 2009, similar total cost estimates, as compared with the overall patient group, were observed in patients in CKD stage 4 or 5 and on haemodialysis and peritoneal dialysis, while transplanted patients who received a kidney transplant in 2009 had considerably higher costs (see etables 4 and 5).

In sensitivity analysis, where we in addition to age, sex and diabetes status adjusted for malignancy, circulatory disease, hypertension, cardiovascular disease, myocardial infarction, stroke and chronic obstructive pulmonary disease, similar mean annual cost ratio estimates as compared with the main analysis were observed. Likewise, when we fitted a generalised linear model with log- $\gamma$ distribution, instead of using a negative binomial distribution, the estimates were similar between the models.

\section{Drug costs}

Prescription drug costs

The mean annual cost of dispensed prescription drugs was $€ 7400$ in the peritoneal dialysis group, €6800 in transplanted patients, $€ 8400$ (of which $€ 3911$ on ESA) in patients on haemodialysis and $€ 2900$ in the CKD 4 or 5 group (table 2, figure 3 ).

\section{Costs related to fluids in peritoneal dialysis}

Based on a regional report of dialysis care in southern Sweden, the annual cost of fluids in patients on peritoneal dialysis was estimated at $€ 29900$. $^{31}$

\section{Healthcare use}

Annual hospital days

Mean annual hospital days in patients on haemodialysis were 21.4, with the majority of hospital days listing a main or contributory diagnosis for cardiovascular disease (8.2 days) or infection (6.7 days; table 2). Patients on peritoneal dialysis displayed lower overall mean hospital days (16.0), with 5.4 and 2.6 days related to cardiovascular disease and infection, respectively. Transplanted patients and patients in CKD stage 4 or 5 had similar level of inpatient care use with 4.6 and 6.2 annual hospital days, respectively.

\section{Outpatient care}

In patients on haemodialysis, 152 out of a mean 159 annual visits were due to dialysis. The corresponding 

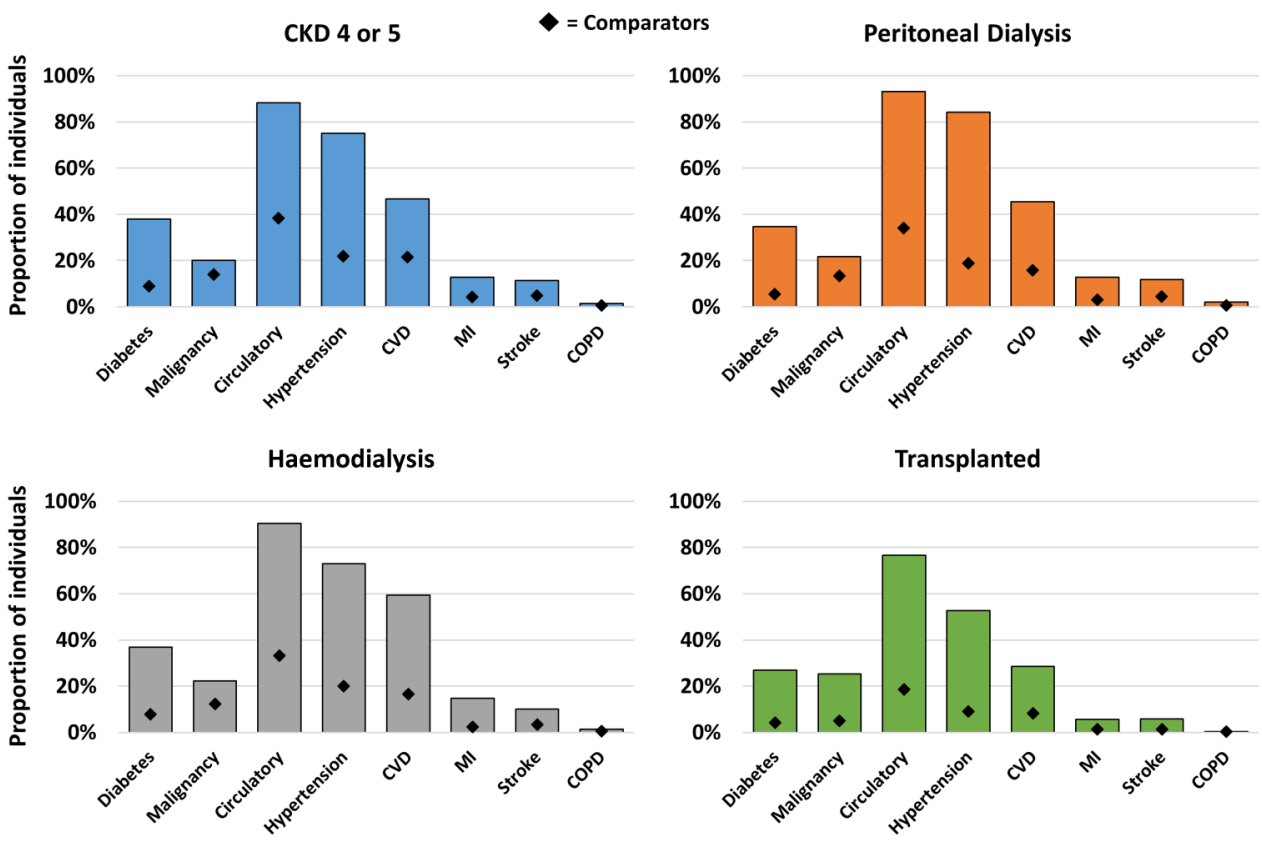

Figure 2 Comorbidity status in patients with CKD stage 4 or 5 not on dialysis, peritoneal dialysis, haemodialysis and transplanted patients (bars), as well as in their matched general population comparators (black diamonds). Comorbid conditions defined as having a visit in inpatient or outpatient care during the past 10 years with a main or contributory diagnosis of the respective ICD-codes used (specified in etable 1). CKD, chronic kidney disease; COPD, chronic obstructive pulmonary disease; CVD, cardiovascular disease; ICD, International Classification of Diseases; MI, myocardial infarction.

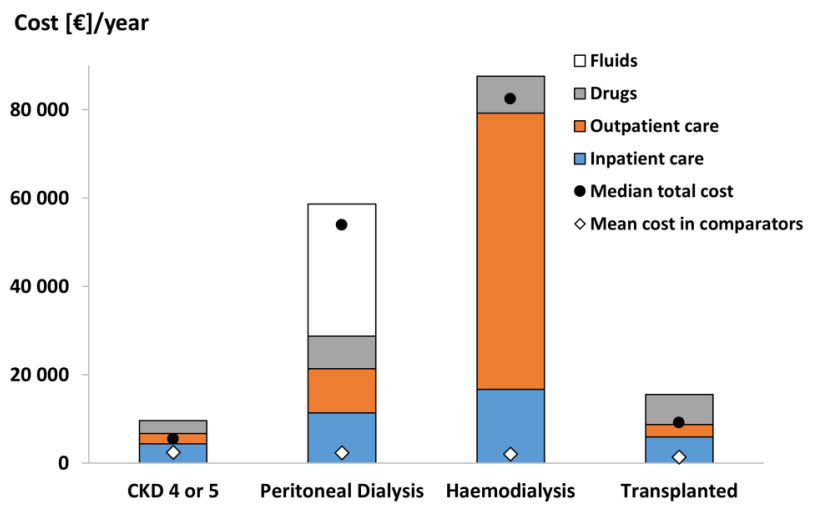

Figure 3 Annualised mean and median costs in patients with CKD stage 4 or 5 not on dialysis, peritoneal dialysis, haemodialysis and transplanted patients, as well as mean total cost in matched general population comparators (matched 5:1 by age, sex and index year). CKD, chronic kidney disease.

number in patients on peritoneal dialysis was 29 visits per year with 15 visits listing dialysis. Again, transplanted patients and patients in CKD stages 4 or 5 had similar numbers with 10 and 8 visits, respectively.

\section{Healthcare use and costs compared with the general population}

Mean annual healthcare use in the matched general population comparators to CKD stages 4 or 5 , peritoneal dialysis and haemodialysis patients were $0.2-0.3$ hospital days, 1.8-2.2 outpatient visits and $€ 500-€ 600$ in prescription drug costs, which resulted in a mean annual total cost of €2000-€2400 (figure 3).

The corresponding healthcare use in general population comparators matched to the younger transplanted patients was 0.1 hospital days, 1.4 outpatient visits and $\$ 400$ in drug costs, resulting in a mean annual total cost of $€ 1300$.

Compared with their matched general population comparators, the mean annual cost was 45 (95\% CI 39 to 51) times higher in the haemodialysis group, 29 (95\% CI 22 to 37) times higher in the peritoneal dialysis group, 11 (95\% CI 10 to 13) times higher in transplanted patients and 4.0 (95\% CI 3.6 to 4.5) times higher in patients with CKD stage 4 or 5 (figure 3 ; table 3 ).

\section{DISCUSSION \\ Principal findings}

In this population-based cohort study, we found that CKD stage 4 or 5 and renal replacement therapy are associated with substantial healthcare costs ranging from 4 to 45 times that expected in the general population. With 3.1 visits per week in outpatient care, patients on haemodialysis had the highest healthcare costs (€87600) with dialysis care making up more than twothirds of the total cost. The total healthcare cost in patients on haemodialysis was about $50 \%$ higher than that in patients on peritoneal dialysis, where similar costs of inpatient and outpatient care were observed, while cost of fluids was a major cost driver in peritoneal dialysis $(€ 29900)$. The mean annual costs in transplanted 
Table 2 Mean (SD) annualised hospital admission, outpatient visits and dispensed prescription drug costs (ICD and ATC codes are specified in etable 1)

\begin{tabular}{|c|c|c|c|c|}
\hline & $\begin{array}{l}\text { CKD stage } 4 \text { or } 5 \\
\text { (not on dialysis) }\end{array}$ & $\begin{array}{l}\text { Peritoneal } \\
\text { dialysis }\end{array}$ & Haemodialysis & Transplanted \\
\hline Annual admissions & $1.0(1.9)$ & $2.6(3.3)$ & $3.6(3.8)$ & $0.9(1.7)$ \\
\hline Annual hospital days & $6.2(16.4)$ & $16.0(25.1)$ & $21.4(40.6)$ & $4.6(11.5)$ \\
\hline Cardiovascular disease & $2.3(8.3)$ & $5.4(15.1)$ & $8.2(29.1)$ & $0.7(3.7)$ \\
\hline Malignancy & $0.5(3.8)$ & $0.3(2.2)$ & $0.7(7.1)$ & $0.2(2.9)$ \\
\hline Infection & $1.1(5.2)$ & $2.6(7.5)$ & $6.7(21.6)$ & $1.5(6.6)$ \\
\hline Annual outpatient visits & $7.8(6.8)$ & $28.6(19.4)$ & $159.4(28.8)$ & $9.6(10.5)$ \\
\hline Dialysis & 0 & $15.2(18.5)$ & $152.2(27.4)$ & $0.1(0.7)$ \\
\hline Cardiovascular disease & $0.3(1.1)$ & $0.3(1.2)$ & $0.3(0.7)$ & $0.2(0.7)$ \\
\hline Malignancy & $0.4(2.0)$ & $0.3(1.2)$ & 0.5 (3.3) & $0.4(2.6)$ \\
\hline Prescription drug costs, $€$ & 2917 (3690) & $7353(6870)$ & 8395 (4286) & $6794(5134)$ \\
\hline $\mathrm{ESA}^{*}$ & 957 (1805) & $2913(2510)$ & 3911 (938) & 268 (952) \\
\hline Antineoplastic and immunomodulating agents & $189(1105)$ & $97(449)$ & $249(973)$ & $5137(4115)$ \\
\hline Various & $297(1675)$ & $1086(1357)$ & $1420(1460)$ & $23(161)$ \\
\hline Cardiovascular system & $434(410)$ & 399 (459) & $285(329)$ & 371 (327) \\
\hline Antidepressant drugs & $54(330)$ & $42(179)$ & $68(171)$ & $30(177)$ \\
\hline Fluids for peritoneal dialysis, $\dagger €$ & - & 29900 & - & - \\
\hline
\end{tabular}

${ }^{*}$ For haemodialysis, costs related to ESA were estimated from a smaller sample $(n=85)$ of patients on haemodialysis as ESA use in this patient group does not enter the Prescribed Drug Register as it is administrated in the hospital in conjunction with dialysis. †Based on a regional report of dialysis care in southern Sweden. ${ }^{31}$

ATC, Anatomic Therapeutic Chemical; CKD, chronic kidney disease; ESA, Erythropoietin stimulating agents; ICD, International Classification of Diseases.

patients was $€ 15500$, where prescription drugs constituted almost $50 \%$, and in CKD stage 4 or $5 € 9600$, with inpatient care making up almost half of the total cost.

\section{Strengths and limitations}

The strengths of this study include the population-based data collected from routine clinical care in the Swedish healthcare system where registered individuals in Sweden have universal access. No comorbidity-based inclusion restrictions were used. Instead, we reported comorbidity status among included patients, as well as prescription drug costs in drug groups, and costs of hospital admissions and outpatient visits for specific diagnoses (eg, cardiovascular disease). Furthermore, using individual-level data from several nationwide registers, linked together by using the personal identity number, follow-up was virtually complete regarding outcome data for hospital days, hospital-based outpatient visits and prescription drug cost, as well as data on comorbidities, death and migration. Available registers enabled analysis of healthcare use in CKD stage 4 or 5 , as well as in patients on haemodialysis, peritoneal dialysis and transplanted patients during the same year and at the same hospitals using the same data sources. Finally, we had the possibility to match comparators from the general population to each patient as a benchmark for healthcare resource usage.

One limitation was that not all healthcare cost components were included in the available register sources. Fluids used for patients on peritoneal dialysis were not included in the Prescribed Drug Register, and these costs were therefore assessed using aggregated data. ${ }^{31}$ Similarly, costs related to ESA for patients on haemodialysis are usually administered in the hospital, resulting in individual-level data for ESA not being recorded in the Prescribed Drug Register. Using a smaller sample of patients on haemodialysis, we could assess the costs related to ESA and apply this estimate to all patients on haemodialysis in the study. Applying these aggregated costs to all patients on peritoneal dialysis (fluids) and

Table 3 Adjusted cost ratios* $(95 \% \mathrm{Cl})$ of annualised total costs between patients with CKD stage 4 or 5 not on dialysis, peritoneal dialysis, haemodialysis and transplanted patients, and their matched general population comparators

\begin{tabular}{llllll}
\hline & $\begin{array}{l}\text { CKD stage } 4 \text { or } 5 \\
\text { (not on dialysis) }\end{array}$ & $\begin{array}{l}\text { Peritoneal } \\
\text { dialysis }\end{array}$ & Haemodialysis & Transplanted & $\begin{array}{l}\text { General } \\
\text { population }\end{array}$ \\
\hline CKD 4 or 5 & - & $0.15(0.14$ to 0.17$)$ & $0.11(0.10$ to 0.11$)$ & $0.59(0.52$ to 0.66$)$ & $4.0(3.6$ to 4.5$)$ \\
Peritoneal dialysis & $6.45(5.81$ to 7.17$)$ & - & $0.67(0.62$ to 0.73$)$ & $4.04(3.58$ to 4.56$)$ & $28.5(21.8$ to 37.4$)$ \\
Haemodialysis & $9.41(8.74$ to 10.1$)$ & $1.49(1.38$ to 1.60$)$ & - & $5.97(5.49$ to 6.50$)$ & $44.5(38.5$ to 51.4$)$ \\
Transplanted & $1.70(1.51$ to 1.92$)$ & $0.25(0.22$ to 0.28$)$ & $0.17(0.15$ to 0.18$)$ & - & $11.1(9.7$ to 12.7$)$ \\
\hline
\end{tabular}

${ }^{*}$ Adjusted for age, sex and diabetes status.

CKD, chronic kidney disease. 
haemodialysis (ESA) will most likely result in smaller variation and overestimated precision of the cost estimates than would have been observed in data including these cost components. Other costs not included were costs related to primary care and certain laboratory services, probably leading to an underestimation of the actual cost. Furthermore, patients on haemodialysis with few registered dialysis visits were scrutinised using the patient registration databases at the clinics. While a few patients for some reason had apparent missing data on dialysis visits also in this system, we imputed data with the mean. However, we cannot know whether haemodialysis visits were missing in the patients who were not scrutinised, which may result in an underestimation of the true haemodialysis cost.

Although all patients on renal replacement therapy in Stockholm County were included, and the proportion of individuals with undiagnosed CKD stage 4 or 5 may have decreased with time, some patients are identified at the start of dialysis, or die before identification. An unknown number of these individuals were missed, and our results should only be generalised to patients in CKD stage 4 or 5 in nephrology care. Finally, an important underlying assumption with the methodology used in this study, where a mix of patients with short and long treatment durations was included, is that the proportion of patients starting their treatment has to be constant over time, in this case by calendar year. This assumption is particularly important in transplanted patients who have a high initial cost with the kidney transplant procedure.

\section{Previous research}

A recent study from the UK (costs in UK£2011) recruited 7246 patients with CKD or patients who were receiving dialysis in Europe, North America and Australasia, and hospital admissions were recorded every 6 months at clinic visits. ${ }^{8}$ It reported a mean hospital cost (drug cost not included) in patients with CKD 4 of $£ 3700$, £12 952 in CKD 5, and £20 511 in patients on dialysis. Excluding drug costs, our estimates of $€ 6700$ in CKD stage 4 or 5 and $€ 21400$ in patients on peritoneal dialysis are in line with the UK study. Although the majority of patients in their dialysis group were patients on haemodialysis $(83 \%)$, our cost estimates are higher when combining the haemodialysis and peritoneal dialysis groups (€68 800), mainly due to different methodologies for collecting costs related to dialysis sessions in outpatient care. In our study, we used prospectively recorded visit data from outpatient care, or annualised estimates for those who did not have a full year of follow-up, while they assumed thrice weekly haemodialysis sessions using $£ 25000$ as a per patient annual cost. Our estimated 2.9 dialysis outpatient visits per week (and additional dialysis sessions in inpatient care for some patients), resulted in a substantially higher annual cost of dialysis delivered in the outpatient setting (€60 400), indicating challenges when comparing study results between different healthcare settings.
Cost ratios of haemodialysis and peritoneal dialysis have recently been reported in a comprehensive review including 78 publications from 46 countries. ${ }^{5}$ On the basis of 37 studies between 1998 and 2011 from Europe, the authors conclude that haemodialysis is $30-60 \%$ more expensive than peritoneal dialysis, while the cost ratio based on five studies from the USA from 2005 to 2012 was estimated at 1.29 , results that are less than our estimated ratio (adjusted cost ratio 1.49 (95\% CI 1.38 to 1.60)).

Recent estimates (2010) from the USA have reported a mean annual healthcare cost of US\$12 386 in patients in CKD $4(\mathrm{n}=413)$ and US\$23 445 in patients in CKD 5 $(\mathrm{n}=138)$ using health insurance claims data. ${ }^{10}$ These estimates are higher than our result in the CKD 4 or 5 group (€9600). Although both studies used the same eGFR intervals when defining CKD stages 45 , our result was more similar to their estimated mean annual cost in patients in CKD 3 (US\$10 100). The differences appeared to be driven by a 2-3 times higher cost of outpatient care in CKD stages 4 and 5 and 3 times higher cost of inpatient care in patients in CKD 5 in their study, while our drug cost estimates were higher.

Another study from the USA in 2004 reported a mean annual cost in patients in CKD stage $4(n=777)$ of US $\$ 7600$, which was 2.6 times the cost as compared with age-matched and sex-matched controls without CKD, results that are in line with our estimates when restricting the CKD 4 or 5 group to CKD stage 4 only ( $€ 8500$ and 3.5 times the cost of comparators).

With respect to previous cost estimates from Sweden, a regional study from 2002, based on questionnaires in 136 patients, estimated the per patient mean annual cost related to peritoneal dialysis at US\$34 600 and in haemodialysis at US\$36 220 during the first 5 years after initiating treatment. ${ }^{18}$ When taking inflation into account, our estimates are substantially higher, which may partly be explained by increased costs due to technology development of medical equipment (their estimates were based on data from 1990 to 1993), and by more complete follow-up when using register data. However, when comparing our adjusted mean annual cost ratio of haemodialysis and peritoneal dialysis, the results are similar.

\section{Implications}

With the lower cost in CKD stage 4 or 5 as compared with dialysis, our result highlights the importance of good secondary prevention of patients in CKD stage 4 or 5 to postpone or even prevent the progression to endstage renal disease, a strategy that may generate significant savings, while also reducing the risk of mortality among these patients. ${ }^{2}$

\section{CONCLUSION}

The annual healthcare costs in patients in CKD stage 4 or 5, in dialysis or transplanted patients are substantial. Patients on haemodialysis incurred the highest cost, 45 
times as compared with the general population, and $50 \%$ higher than patients on peritoneal dialysis. Transplanted patients and patients in CKD stage 4 or 5 incurred lower but considerable costs with 11 and 4 times the cost in the general population, respectively. More attention to secondary prevention in CKD stage 4 or 5 may generate savings by reducing the time and number of patients on dialysis.

\section{Author affiliations}

${ }^{1}$ Clinical Epidemiology Unit, Department of Medicine, Karolinska Institutet, Stockholm, Sweden

${ }^{2}$ Division of Nephrology, Department of Clinical Sciences, Danderyd University Hospital, Stockholm, Sweden

${ }^{3}$ Nephrology Unit, Department of Clinical Sciences, Intervention and Technology, Stockholm, Sweden

${ }^{4}$ Unit for Evidence Based Medicine, Stockholm County Council, Stockholm, Sweden

${ }^{5}$ Unit of Renal Medicine, Department of Medicine, Karolinska Institutet, Stockholm, Sweden

Acknowledgements The authors would like to give a special thanks to Nina Janson-Broström for valuable input and quality control of haemodialysis visits at Karolinska University Hospital, and to Jeanette Wallin for the work of verifying haemodialysis visits at Danderyd University Hospital.

Contributors BH, C-GE, JKE and MN conceived the study hypothesis. JKE conducted the statistical analyses. JKE wrote the first draft of the manuscript. $\mathrm{BH}, \mathrm{C}-\mathrm{GE}, \mathrm{JKE}, \mathrm{MN}$ and SHJ critically reviewed and contributed to the final draft. All authors are guarantors.

Funding This work was supported by Stockholm County Council and Baxter. Statement of independence of researchers from funders $\mathrm{BH}, \mathrm{SHJ}$ and $\mathrm{C}-\mathrm{GE}$ are employed by Stockholm County Council.

Competing interests MN has received payment for a lecture from Baxter. $\mathrm{C}-\mathrm{GE}$ and $\mathrm{BH}$ have received a grant to their academic institution from Baxter to support the work with this publication. SHJ has acted on an advisory board for Baxter, and received lecture payments at scientific meetings.

Ethics approval Ethical approval was granted by the Regional Ethics Committee at Karolinska Institutet, Stockholm, Sweden (DNR: 2009/ $1225-31 / 5)$.

Provenance and peer review Not commissioned; externally peer reviewed.

Data sharing statement No additional data are available.

Open Access This is an Open Access article distributed in accordance with the Creative Commons Attribution Non Commercial (CC BY-NC 4.0) license, which permits others to distribute, remix, adapt, build upon this work noncommercially, and license their derivative works on different terms, provided the original work is properly cited and the use is non-commercial. See: http:// creativecommons.org/licenses/by-nc/4.0/

\section{REFERENCES}

1. Klarenbach SW, Tonelli M, Chui B, et al. Economic evaluation of dialysis therapies. Nat Rev Nephrol 2014;10:644-52.

2. Neovius M, Jacobson SH, Eriksson JK, et al. Mortality in chronic kidney disease and renal replacement therapy: a population-based cohort study. BMJ Open 2014;4:e004251.

3. Collins AJ, Foley RN, Gilbertson DT, et al. United States Renal Data System public health surveillance of chronic kidney disease and end-stage renal disease. Kidney Int Suppl (2011) 2015;5:2-7.

4. Hamer RA, EI Nahas AM. The burden of chronic kidney disease. BMJ 2006;332:563-4.

5. Karopadi AN, Mason G, Rettore E, et al. Cost of peritoneal dialysis and haemodialysis across the world. Nephrol Dial Transplant 2013;28:2553-69.

6. Menzin J, Lines LM, Weiner DE, et al. A review of the costs and cost effectiveness of interventions in chronic kidney disease: implications for policy. Pharmacoeconomics 2011;29:839-61.
7. De Vecchi AF, Dratwa M, Wiedemann ME. Healthcare systems and end-stage renal disease (ESRD) therapies - an international review: costs and reimbursement/funding of ESRD therapies. Nephrol Dial Transplant 1999;14(Suppl 6):31-41.

8. Kent S, Schlackow I, Lozano-Kühne $\mathrm{J}$, et al. What is the impact of chronic kidney disease stage and cardiovascular disease on the annual cost of hospital care in moderate-to-severe kidney disease? BMC Nephrol 2015;16:65.

9. Smith DH, Gullion CM, Nichols G, et al. Cost of medical care for chronic kidney disease and comorbidity among enrollees in a large HMO population. J Am Soc Nephrol 2004;15:1300-6.

10. Vekeman F, Yameogo ND, Lefebvre P, et al. Healthcare costs associated with nephrology care in pre-dialysis chronic kidney disease patients. J Med Econ 2010;13:673-80.

11. Wyld ML, Lee CM, Zhuo X, et al. Cost to government and society of chronic kidney disease stage 1-5: a national cohort study. Intern Med J 2015;45:741-7.

12. Baumeister SE, Böger CA, Krämer BK, et al. Effect of chronic kidney disease and comorbid conditions on health care costs: a 10-year observational study in a general population. Am J Nephrol 2010;31:222-9.

13. Berger A, Edelsberg J, Inglese GW, et al. Cost comparison of peritoneal dialysis versus hemodialysis in end-stage renal disease. Am J Manag Care 2009;15:509-18.

14. Honeycutt AA, Segel JE, Zhuo X, et al. Medical costs of CKD in the Medicare population. J Am Soc Nephrol 2013;24:1478-83.

15. Icks A, Haastert B, Gandjour A, et al. Costs of dialysis-a regional population-based analysis. Nephrol Dial Transplant 2010;25:1647-52

16. Kerr M, Bray B, Medcalf $J$, et al. Estimating the financial cost of chronic kidney disease to the NHS in England. Nephrol Dial Transplant 2012;27(Suppl 3):iii73-80.

17. Lee $\mathrm{H}$, Manns $\mathrm{B}$, Taub K, et al. Cost analysis of ongoing care of patients with end-stage renal disease: the impact of dialysis modality and dialysis access. Am J Kidney Dis 2002;40:611-22.

18. Sennfält K, Magnusson M, Carlsson P. Comparison of hemodialysis and peritoneal dialysis-a cost-utility analysis. Perit Dial Int 2002;22:39-47.

19. Villa G, Rodriguez-Carmona A, Fernández-Ortiz L, et al. Cost analysis of the Spanish renal replacement therapy programme. Nephrol Dial Transplant 2011;26:3709-14.

20. Baboolal K, McEwan P, Sondhi S, et al. The cost of renal dialysis in a UK setting-a multicentre study. Nephrol Dial Transplant 2008;23:1982-9.

21. Salonen $\mathrm{T}$, Reina $\mathrm{T}, \mathrm{Oksa} \mathrm{H}$, et al. Cost analysis of renal replacement therapies in Finland. Am J Kidney Dis 2003:42:1228-38.

22. Ludvigsson JF, Otterblad-Olausson P, Pettersson BU, et al. The Swedish personal identity number: possibilities and pitfalls in healthcare and medical research. Eur J Epidemiol 2009;24:659-67.

23. Wikström B, Fored M, Eichleay MA, et al. The financing and organization of medical care for patients with end-stage renal disease in Sweden. Int $J$ Health Care Finance Econ 2007;7:269-81.

24. Svensk Njurmedicinsk Förening. Riktlinjer för omhändertagande av patienter med njursvikt. Svensk Njurmedicinsk Förening (SNR), 2007. http://www.njur.se/filer/behandlingsriktlinjer/riktlinjer uremi 2007.pdf (accessed 28 Sep 2016)

25. (K/DOQI) KDOQI. K/DOQI clinical practice guidelines on hypertension and antihypertensive agents in chronic kidney disease. Am J Kidney Dis 2004;43(Suppl 1):S1-290.

26. Hemodialysis EBPGEGo. Section V. Chronic intermittent haemodialysis and prevention of clotting in the extracorporal system. Nephrol Dial Transplant 2002;17(Suppl 7):63-71.

27. Levey AS, Stevens LA, Schmid CH, et al. A new equation to estimate glomerular filtration rate. Ann Intern Med 2009;150:604-12.

28. Schön S, Ekberg H, Wikström B, et al. Renal replacement therapy in Sweden. Scand J Urol Nephrol 2004;38:332-9.

29. Svenskt Njurregister. Renal replacement therapy in Sweden 19912009, 2010. http://www.medscinet.net/snr/rapporterdocs/AKTIV\% 20UREMIV\%C3\%85RD\%20I\%20SVERIGE\%201991\%20-\%202009. pdf (accessed 28 Sep 2016).

30. Socialstyrelsen. The National Patient Register. Secondary The National Patient Register, 2011. http://www.socialstyrelsen.se/ register/halsodataregister/patientregistret/inenglish

31. Prütz K. Dialysvården i Skåne. http://www.skane.se/upload/ webbplatser/skaneportalen-extern/vardhalsa/utredningar_rapporter/ dokument/delrapport1_dialysvarden_i_skane.pdf, 2003.

32. Thompson SG, Barber JA. How should cost data in pragmatic randomised trials be analysed? BMJ 2000;320:1197-200. 